\title{
A Simulation Study on Improving Throughput in a Crankshaft Line Considering Minimizing Investment Cost under Throughput Target
}

\author{
Guan Wang, Dae Ryoung Lee, Yang Woo Shin, and Dug Hee Moon
}

\begin{abstract}
In this paper, we consider a problem minimizing investment cost when throughput target is given, and the total budget and available spaces for machines and buffers are limited. This situation happens frequently in practical manufacturing system, and we consider the crankshaft line of a Korean automotive factory as for the case study. Simulation model is developed to estimate throughput and a modified arrow assignment rule using difficulty is suggested to find optimal allocation of machines and buffers.
\end{abstract}

Index Terms-Budget, crankshaft line, hybrid flow shop, throughput.

\section{INTRODUCTION}

Manufacturing system is the organic combination of various types of machines, transportation equipment, storage equipment, and so on. Developing a new manufacturing system needs several iterative processes such as determining the overall concept of layout pattern, selecting the types of machines and material handling equipment, and assigning operations to machines. In design phase, an engineer has interests in the throughput of system and investment costs. Generally, increasing the number of machine and buffer capacity is easy and efficient way to increase the throughput, but it requires the investment cost, inventory holding cost and space cost. Thus, there is a trade-off between throughput and costs.

In design phase, buffer allocation scheme is directly related to the performances of manufacturing system which include throughput, work in process (WIP) and profit et al. The buffer allocation scheme should be modified whenever the layout structure is changed from an existing system. Therefore, buffer allocation problem is very important and there have been many researches dealing with solving this problem. For the flow manufacturing lines with single

Manuscript received March 1, 2015; revised October 16, 2015. This work was supported by Basic Research Program through the National Research Foundation of Korea (NRF) funded by the Ministry of Education (Grant Numbers NRF-2013R1A1A2058943).

G. Wang is with the School of Management and Economics, Hebei University of Science and Technology, Shijiazhuang City, Hebei Province, China PR (e-mail: wgcan1@hotmail.com).

D. R. Lee is with Department of Eco-friendly Marine Plant FEED Engineering, Changwon National University, Changwon, Gyeongnam, 641-773, Korea (e-mail: dudtlrdl2@naver.com).

Y. W. Shin is with the Department of Statistics, Changwon National University, Changwon, Gyeongnam, 641-773, Korea (e-mail: ywshin@changwon.ac.kr).

D. H. Moon is with Department of Industrial and Systems Engineering, Changwon National University, Changwon, Gyeongnam, 641-773, Korea (corresponding author; e-mail: dhmoon@changwon.ac.kr). reliable machine, Powell [1] suggested the rules of thumb for the optimal sequential placement of buffer space in the unbalanced lines with three machines. Huang et al. [2], Diamantidis and Papadopoulos [3] used the dynamic programming approach to maximize the production rate or minimize the work in process under a certain buffer allocation strategy in the flow shop-type manufacturing systems. Chan and Ng [4] compared buffer allocation strategies for maximizing the production rate. Papadopoulos et al. [5] compared five different optimize approaches for maximizing the throughput, minimizing the idle time and WIP.

Only few researchers considered about the flow manufacturing line with parallel unreliable machines, e.g. Dolgui et al. [6] used the genetic algorithm to maximize the profit. Actually, the engineers prefer to solve the buffer allocation problem and make a procurement scheme for additional machines at the same time. Because it often seems that just re-allocate the buffer spaces is powerless to meet the target and they have to consider the buffer allocation scheme again after purchasing additional machines. Nahas et al. [7] adopted colony optimization for selecting machines and buffers under constrain of total investment cost to maximize the throughput. However, the limitation of mathematical approximation method, which is adopted to measure the performances of manufacturing lines in their papers, is that mathematical model cannot complete reflect the characteristics of complex manufacturing line such multiple failure rate and defective detection et al. Thus, simulation models are used for estimating the performances of manufacturing lines.

Although the simulation models can be adopted to estimate the performances of complex manufacturing line, computation speed is a fatal weakness. Thus, we need faster heuristic and reliable algorithm. Bottleneck detection method is an important tool to speed up the search process. There are some algorithms to find the bottleneck in a serial line, e.g., “Arrow Assignment Rule: AAR” (see Li and Meerkov [8]; Li et al. [9]), “Active Period Method” (see Kwon and Lim [10]) and a method using autoregressive moving average (ARMA) model (see Li et al. [11]). In Arrow Assignment Rule, a serial line having only one machine in each stage which has one type of time dependent failure is considered and mathematical approximation model for estimating throughput, WIP, blocking probabilities and starving probabilities is presented.

Wang et al. [12] discussed a problem for improving the throughput of a crankshaft manufacturing line in an 
automotive factory in which the budget for purchasing new machines and installing additional buffers is limited. They also considered the constraint of available space for both of machine and buffer.

This paper is an extension of Wang et al. [12] in a way that the objective function is to minimize investment cost and there is a throughput target is determined. We suggests a method to solve the machine selecting and buffer allocation problem at the same time to meet the increasing target of throughput using the minimized investment cost under the constrain of limited investment budget. In practice, three types of strategies are usually applied to increase throughput, and they are buying additional machines, installing additional buffers and replacing tools with longer life cycles. However, in this paper we only consider the strategy of buying new machines and adding buffers.

\section{Problem StATEMENTS}

The objective function is to minimize total investment cost considering the throughput target and shown as follow:

$$
\begin{gathered}
\min \operatorname{Inv}=\sum_{i=1}^{N} C_{i} x_{i}+\sum_{i=1}^{N-1} D_{i} y_{i} \\
\text { s.t. } \sum_{i=1}^{N} C_{i} x_{i}+\sum_{i=1}^{N-1} D_{i} y_{i} \leq B \\
\Phi\left(x_{1}, . ., x_{N}, y_{1}, . ., y_{N-1}\right)>T P_{t \text { arg } e t} \\
x_{i} \text { : integer and } 0 \leq x_{i} \leq f_{i} \text { for } i=1, \ldots, N, \\
y_{i} \text { : integer and } 0 \leq y_{i} \leq g_{i} \text { for } i=1, \ldots, N-1,
\end{gathered}
$$

where $x_{i}$ and $f_{i}$ are the number of additional machines and its upper bound in operation $i$, respectively. $y_{i}$ and $g_{i}$ are the number of additional buffer and its upper bound between operations $i$ and $i+1$. Furthermore, $\Phi$ is the throughput of the system, $C_{i}$ is the price of machine $i, D_{i}$ is the cost of additional one buffer and $B$ is the total budget available. $T P_{\text {target }}$ means the expected target of throughput.

\section{HEURISTIC SOLUTION AlgORITHM}

In this paper, we adopt the concept of AAR for finding bottleneck and modify it with the considerations of limited budget and extra spaces for machines and buffers. In AAR, the severity $\left(S_{i}\right)$ which is used for finding bottleneck is defined as follows.

$$
\begin{gathered}
\mathrm{S}_{\mathrm{i}}=\left|S T_{i+1}-B L_{i}\right|+\left|S T_{i}-B L_{i-1}\right|, \text { for } i=2, \ldots, N-1 \\
\mathrm{~S}_{1}=\left|S T_{2}-B L_{1}\right| \\
\mathrm{S}_{\mathrm{N}}=\left|S T_{N}-B L_{N-1}\right|,
\end{gathered}
$$

where $B L_{i}$ and $S T_{i}$ the blocking probability of machine $i\left(m_{i}\right)$ and the starving probability of $m_{i}$ in steady state, respectively. Then, $S_{i}$ of $m_{i}$ is defined as in equation (6).
If $B L_{i}>S T_{i+1}$, assign the arrow pointing from $m_{\mathrm{i}}$ to $m_{\mathrm{i}+1}$. If $B L_{i}<S T_{i+1}$, assign the arrow pointing from $\mathrm{m}_{i+1}$ to $\mathrm{m}_{i}$. In case that there are multiple machines with no emanating arrows, the one with the largest severity $\left(S_{i}\right)$ is primary.

However, AAR is designed for finding bottleneck in a flow line and it does not consider investment cost. Thus, we introduce a new criterion to determine the priority of investment considering investment cost as shown in equations (9), which is called as the difficulty.

$$
\text { Difficulty }=\frac{T P_{t \arg e t}-\Phi\left(x_{1}, . ., x_{N}, y_{1}, . ., y_{N-1}\right)}{\Delta I n v}
$$

where $\Delta I n v$ is the increment of investment cost.

Then, the solution algorithm is depicted as in Fig. 1, and the notations used in Fig. 1 are as follows.

- TP: throughput,

- $B N$ : set of bottleneck machines,

- COM: machine candidate,

- COB: buffer candidate,

- $d(C O M)$ : difficulty of machine $C O M$,

- $d(C O B)$ : difficulty of buffer $C O B$,

- $p \_B N \_m$ : primary bottleneck machine having the largest $S_{i}$ in $B N$,

- $s \_B N \_m$ : set of secondary bottleneck machines having the smaller $S_{i}$ than $p \_B N \_m$ in $B N$,

- $s \_B N \_m \_$avl: subset of available machines in $s \_B N \_m$,

- $s \_B N \_m^{*}$ : machine having the largest in $s \_B N \_m \_a v l$,

- $p \_B N \_b$ : primary bottleneck buffer, where

$$
p_{-} B N_{-} b=\left\{\begin{array}{l}
\text { Upbuffer of } p_{-} B N_{-} m, \text { if } S T_{i}>B L_{i} \\
\text { Down buffer of } p_{-} B N_{-} m, \text { if } S T_{i}<B L_{i},
\end{array}\right.
$$

- $s \_B N \_b i$ : set of secondary bottleneck buffer related to each machine $i$ in $s \_B N \_m$, where

$$
s_{-} B N_{-} b_{i}=\left\{\begin{array}{l}
\text { UP buffer of } p_{-} B N_{-} m_{i}, \text { if } S T_{i}>B L_{i} \\
\text { DOWN buffer of } p_{-} B N_{-} m_{i}, \text { if } S T_{i}<B L_{i}
\end{array},\right.
$$

- $s \_B N \_b \_a v l$ : subset of available buffers in $s \_B N \_b$,

- $s \_B N \_b^{*}$ : the buffer related to the machine having the largest $S i$ in $s \_B N \_m$,

$$
B N_{-} \text {Side }=\left\{\begin{array}{l}
U P \text { side of } p_{-} B N_{-} m, \text { if } S T_{i}>B L_{i} \\
D O W N \text { side of } p_{-} B N_{-} m, \text { if } S T_{i}<B L_{i},
\end{array}\right.
$$

- $B N \_s i d e \_m:$ set of machines on $B N \_s i d e$, which are not included in $B N$,

- BN_side_m_avl: subset of available machines in $B N \_s i d e \_m$,

- BN_side_m*: machine having the largest $S i$ in BN_side_m_avl,

- $B N \_$side_b: set of buffers on $B N \_$side which are neither $p \_B N \_b$ nor the buffers in $s \_B N \_b$,

- BN_side_b_avl: subset of available buffers in $B N \_s i d e \_b$,

- $B N \_s i d e \_b^{*}$ : the buffer related to the machine having the largest severity in $B N \_s i d e \_m$,

$$
\text { nonBN_Side }=\left\{\begin{array}{l}
\text { Up side of } p_{-} B N_{-} m, \text { if } S T_{i}>B L_{i} \\
\text { Down side of } p_{-} B N_{-} m, \text { if } S T_{i}<B L_{i}
\end{array},\right.
$$


- non $B N \_s i d e \_m$ : set of machines on non $B N \_$side, which are not included in $B N$,

- non BN_side_m_avl: subset of available buffers in non $B N \_s i d e \_m$,

- non BN_side_m*: machine having the largest in non BN_side_m_avl,

- non $B N \_s i d e \_b$ : set of buffers on non $B N \_$side which are neither $p \_B N \_b$ nor buffers in $s \_B N \_b$,

- non BN_side_b_avl: subset of available buffers in non BN_side_b,

- non $B N \_$side_ $b^{*}$ : the buffer related to the machine having the largest in non $B N \_s i d e \_m$,

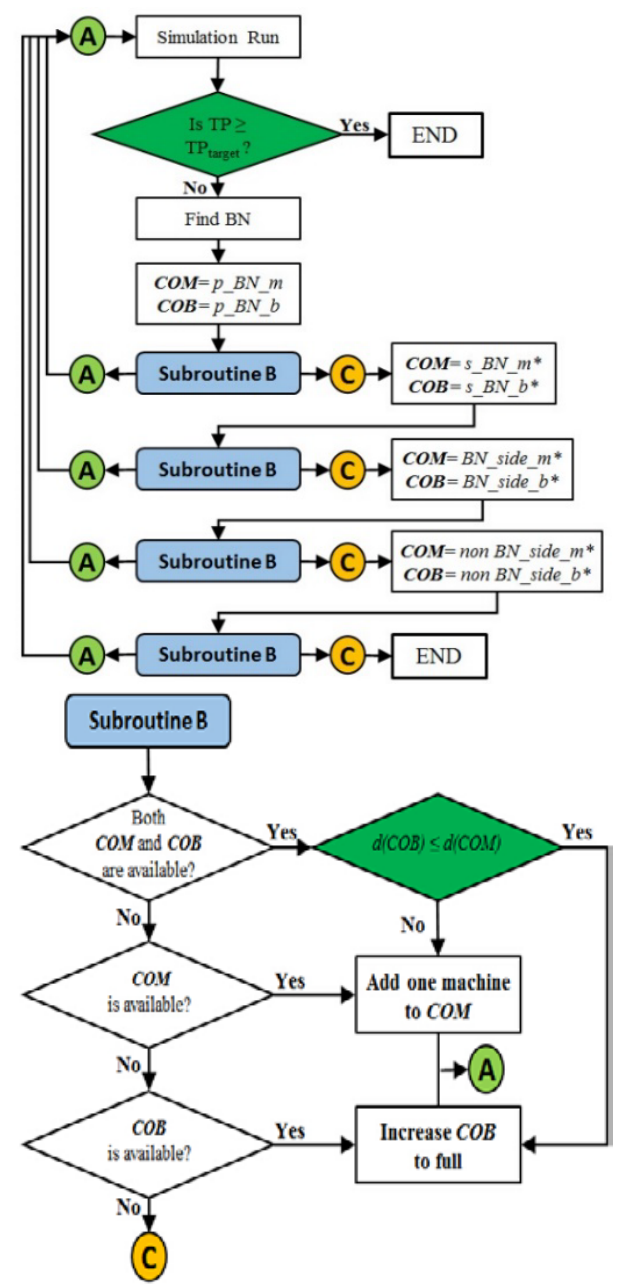

Fig. 1. Flow chart of algorithm suggested.

As shown as in Fig. 1, the search process starts from the simulation run for the As-Is model. Then, the data of throughput, WIP (work in process) starving rate and blocking rate can be collected. Next step, we should use these data to calculate the severity for each operation. After that, $\boldsymbol{B N}$, $p \_B N \_m, p \_B N \_b$ and all others mentioned above can be certain. Until now, $C O B$ and $C O M$ should be selected. The first candidates for $C O B$ and $C O M$ are $p \_B N \_m$ and $p \_B N \_b$. If both of them are available, an additional machine would be added and the $C O B$ would be increase to full respectively. Then, we need to compare the investment difficulty of COM and $C O B$ and select the lower one to be the new element of our plan. If only one of $C O M$ and $C O B$ is available, we just select it to add additional equipment. If they are all unavailable, we should look for available candidates in secondary bottleneck, $B N$ side and non $B N$ side sequentially, until the decision is made.

Then, the heuristic search process would be end when there are no available $C O B$ (candidate of buffer), $C O M$ (candidate of machine), or the throughput reaches target. In other word, that process could be end by three situations. The first is when the remaining budget is not enough to purchase any additional equipment for buffer or machine. The second is there is no available space for additional buffer or machine. The last one is the throughput meets or exceeds the throughput target.

\section{CASE Study}

\section{A. Description of Case Study}

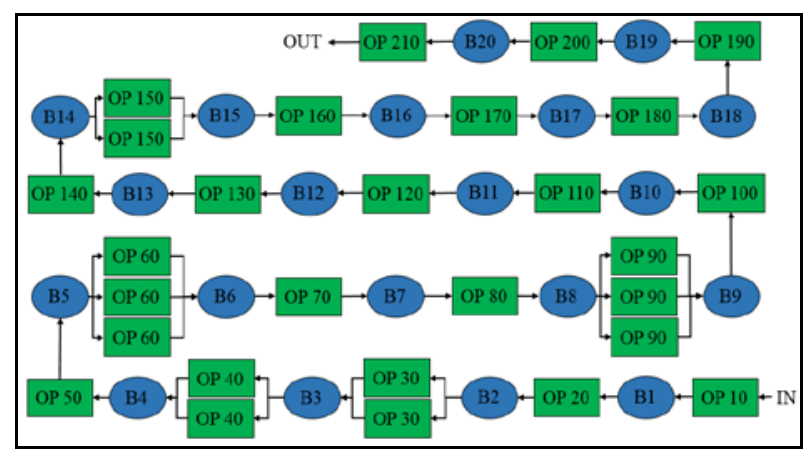

Fig. 2. Processes of crankshaft manufacturing (As-Is).

The suggested algorithm is applied to a crankshaft manufacturing line of a Korean automotive factory as depicted in Fig. 2. We used a same example in Wang et al. [12]. The factory has a plan to increase the production capacity with the smallest budget to meet the increasing demand, and thus, it is necessary to find the bottleneck for growing up throughput.

\begin{tabular}{ccccc}
\multicolumn{5}{c}{ TABLE I: DATA OF OPERATIONS (As-Is) } \\
OP. No. & $\begin{array}{c}\text { Number } \\
\text { of } \\
\text { Machines }\end{array}$ & $\begin{array}{c}\text { Cycle Time } \\
\text { (sec.) }\end{array}$ & $\begin{array}{c}\text { Machine } \\
\text { Price } \\
(\$ 1,000)\end{array}$ & $\begin{array}{c}\text { Extra } \\
\text { Available } \\
\text { Spaces }\end{array}$ \\
\hline OP-10 & 1 & 50 & 1,180 & 0 \\
OP-20 & 1 & 46 & 230 & 0 \\
OP-30 & 2 & 140 & 952 & 1 \\
OP-40 & 2 & 152 & 1,012 & 1 \\
OP-50 & 1 & 50 & 962 & 0 \\
OP-60 & 3 & 195 & 357 & 2 \\
OP-70 & 1 & 48 & 120 & 0 \\
OP-80 & 1 & 51 & 1,010 & 0 \\
OP-90 & 3 & 198 & 357 & 2 \\
OP-100 & 1 & 48 & 270 & 1 \\
OP-110 & 1 & 75 & 833 & 1 \\
OP-120 & 1 & 52 & 1,190 & 1 \\
OP-130 & 1 & 47 & 476 & 1 \\
OP-140 & 1 & 54 & 476 & 1 \\
OP-150 & 2 & 160 & 417 & 2 \\
OP-160 & 1 & 48 & 726 & 0 \\
OP-170 & 1 & 48 & 350 & 0 \\
OP-180 & 1 & 50 & 500 & 0 \\
OP-190 & 1 & 48 & 370 & 0 \\
OP-200 & 1 & 50 & 350 & 0 \\
OP-210 & 1 & 51 & 390 & 0 \\
\hline \hline
\end{tabular}

In this paper, we combine the simulation study for analysing manufacturing system and the bottleneck search method to determine investment plan considering the 
limitation of budget and available spaces for machines and buffers. The simulation model is developed with ARENA ${ }^{\circledR}$.

The layout concept of the crankshaft line considered in this paper is a typical flow line as shown in Fig. 2. In order to enhance the ease of machining or to reduce the risk of the breakdown of a line, some operations have two or three identical machines in parallel where a part chooses only one of machines and then it goes to the next operation after operation. Here, OP-30, OP-40, OP-60, OP-90 and OP-150 consist of multiple identical machines in parallel.

Table I lists the data related to machine and Table II shows the data related to buffers. The current annual production quantity is 87,984 units and the company wants to increase $6 \%$ of the throughput. The price of additional one buffer (jig) is $\$ 200$, and the total investment cost for all additional buffers is $\$ 200 * 155=\$ 31,000$. The maximum budget available is $\$ 1,050,000$. We assumed that the buffer increment is nothing or full. We also considered the failures of machines. Tool change times and defect rates (see Wang et al. [12] for detail data).

TABLE II: DATA OF BUFFERS

\begin{tabular}{ccc}
\hline \hline Buffers & Existing Capacity & Extra Capacity \\
\hline B1 & 20 & 10 \\
B2 & 17 & 13 \\
B3 & 2 & 0 \\
B4 & 17 & 0 \\
B5 & 20 & 10 \\
B6 & 23 & 7 \\
B7 & 15 & 15 \\
B8 & 17 & 13 \\
B9 & 17 & 0 \\
B10 & 20 & 10 \\
B11 & 34 & 0 \\
B12 & 20 & 10 \\
B13 & 17 & 13 \\
B14 & 20 & 10 \\
B15 & 20 & 10 \\
B16 & 1 & 0 \\
B17 & 23 & 7 \\
B18 & 39 & 0 \\
B19 & 16 & 14 \\
B20 & 17 & 13 \\
\hline \hline
\end{tabular}

\section{B. Result and Validation}

Table III shows the solution procedure. The searching process is repeated until the ninth round and the size of B6, B1, B5 and B7 are selected to sequentially increase to full. In the final round, the throughput increases to 93,294, the increment is 5310 units (6.04\%) and WIP is 140.38 . The total investment cost is $\$ 789,400$ and the remaining budget is $\$ 260,600$.

The number of simulation experiments including As-Is analysis is 13 and the average simulation run time for each experiment is about 15 minutes. In rounds 1,3 and 4 we conduct two simulation runs for determining investment plans. To validate the simulation model developed, simulation run time was set to 146,410 minutes including 13,310 minutes of warm up time, and the number of replications was set to 100 . Then, the data gathering time was 133,100 minutes, and it was the 10 months in practice.

To validate the solution procedure suggested, the best solution obtained is compared with the feasible solutions obtained from all enumerations. However, the number of all enumerations is too much big when we assume that the increment size of buffer is set to one. Thus, we inevitably assume that all available buffers are full, and search for the feasible investment plans for machines. Then the number of feasible plans is 30 for $\$ 1,050,000$ budget scenarios as shown in Table IV. If we assume that the capacities of some buffers remain without increasing, then the number of feasible plans must be greater than 30 . We can find that there are six plans exceed the throughput target. Scenario 4 is the best because the investment of it is less than other under the constraint condition which is the throughput increment should be great or equal to $6 \%$. The investment plan that we obtained from our heuristic method is second-best. However, it is obvious that our investment plan has greater growth prospect than all others if the target is raised. Because there still are seven buffers available for our investment plan. If the target was raised to more than $6.10 \%$, our plan would be undisputed best plan.

TABLE III: SUMMARY OF SOLUTION PROCEDURE

\begin{tabular}{clccc}
\hline \hline Iterations & Investment Plan & $\begin{array}{c}\text { Through } \\
\text { put }\end{array}$ & WIP & $\begin{array}{c}\text { Remaining } \\
\text { Budget }\end{array}$ \\
\hline Round 0 & As-Is & 87,984 & 178.54 & $\$ 1,050,000$ \\
Round 1 & OP-150 & 91,571 & 98.25 & $\$ 633,000$ \\
Round 2 & OP-150 B10 & 91,707 & 100.50 & $\$ 631,000$ \\
Round 3 & OP-150 B10 B8 & 91,798 & 102.01 & $\$ 628,400$ \\
Round 4 & OP-150 B10 B8 B2 & 92,146 & 117.51 & $\$ 625,800$ \\
Round 5 & OP-150 B10 B8 B2 & 92,600 & 117.33 & $\$ 268,800$ \\
& OP-90 & & & \\
Round 6 & OP-150 B10 B8 B2 & 92,792 & 118.83 & $\$ 267,400$ \\
& OP-90 B6 & & & \\
Round 7 & OP-150 B10 B8 B2 & 92,933 & 130.13 & $\$ 265,400$ \\
& OP-90 B6 B1 & & & \\
Round 8 & OP-150 B10 B8 B2 & 93,080 & 133.90 & $\$ 263,400$ \\
& OP-90 B6 B1 B5 & & & \\
Round 9 & OP-150 B10 B8 B2 & 93,294 & 140.38 & $\$ 260,600$ \\
& OP-90 B6 B1 B5 B7 & & & \\
\hline \hline
\end{tabular}

TABLE IV: SIMULATION RESULTS (ALL ENUMERATION)

\begin{tabular}{|c|c|c|c|c|}
\hline \multirow{2}{*}{ Scenario } & \multirow{2}{*}{ Investment Plan } & \multicolumn{2}{|c|}{ Throughput } & \multirow{2}{*}{$\begin{array}{c}\text { Investment } \\
(\$ 1,000)\end{array}$} \\
\hline & & Mean & $95 \%$ CI & \\
\hline 1 & OP-90 OP-150 & 94,017 & \pm 149.2 & 805 \\
\hline 2 & OP-130 OP-150 & 93,613 & \pm 154.1 & 924 \\
\hline 3 & OP140 OP-150 & 93,555 & \pm 143.6 & 924 \\
\hline 4 & OP-100 OP-150 & 93,349 & \pm 132.9 & 718 \\
\hline 5 & OP-150 OP-150 & 93,334 & \pm 153.8 & 865 \\
\hline 6 & OP-60 OP-150 & 93,329 & \pm 146.4 & 805 \\
\hline 7 & OP-150 & 93,114 & \pm 139.3 & 448 \\
\hline 8 & OP-130 OP-140 & 91,769 & \pm 174.3 & 983 \\
\hline 9 & OP-60 OP-140 & 91,494 & \pm 140.8 & 864 \\
\hline 10 & OP-90 OP-140 & 91,492 & \pm 149.2 & 864 \\
\hline 11 & OP-90 OP-130 & 91,391 & \pm 138.7 & 777 \\
\hline$:$ & : & : & : & : \\
\hline 28 & OP-100 & 90,575 & \pm 130.7 & 301 \\
\hline 29 & OP-30 & 90,330 & \pm 112.6 & 983 \\
\hline 30 & OP-90 & 90,208 & \pm 126.4 & 388 \\
\hline
\end{tabular}

\section{CONCLUSION}

In this paper, we discussed a simulation study for improving the throughput of a crankshaft manufacturing line in an automotive factory. The goal is to minimize the investment amount for the production line to meet or exceed a given throughput target with a limitation of budget for 
purchasing new machines and installing additional buffers. To determine the investment plan, we modified arrow assignment rule for considering parallel machines, the budget limitation, the space limitations of machines and buffers and a given throughput target. The best solution by the modified arrow assignment rule was compared to the all enumerations. Although the modified arrow assignment rule does not guarantee the optimality, we obtained the reasonably good solution in the case study.

\section{REFERENCES}

[1] S. G. Powell, "Buffer allocation in unbalanced three-station serial lines," International Journal of Production Research, vol. 32, pp. 2201-2217, 1994.

[2] M. G. Huang, P. L. Chang, and Y. C. Chou, "Buffer allocation in flow-shop-type production system with general arrival and service patterns," Computers \& Operations Research, vol. 29, pp. 103-121, 2002.

[3] A. C. Diamantidis and C. T. Papadopoulos, "A dynamic programming algorithm for the buffer allocation problem in homogeneous asymptotically reliable serial production lines," Mathematical Problems in Engineering, vol. 3, pp. 209-223, 2004.

[4] F. T. S. Chan and E. Y. H. Ng, "Comparative evaluations of buffer allocation strategies in a serial production line," The International Journal of Advanced Manufacturing Technology, vol. 19, pp. 789-800, 2002.

[5] C. T. Papadopoulos, M. E. J. O’Kelly, and A. K. Tsadiras, “A DSS for the buffer allocation of production lines based on a comparative evaluation of a set of search algorithms," International Journal of Production Research, vol. 51, pp. 4175-4199, 2013.

[6] A. Dolgui, A. Eremeev, A. Kolokolov, and V. Sigaev, "A genetic algorithm for the allocation of buffer storage capacities in a production line with unreliable machines," Journal of Mathematical Modelling and Algorithms, vol. 1, pp. 89-104, 2002.

[7] N. Nahas, M. Nourelfath, and M. Gendreau, "Selecting machines and buffers in unreliable assembly/disassembly manufacturing networks," International Journal of Production Economics, vol. 154, pp. 113-126, 2014.

[8] J. S. Li and S. M. Meerkov, Production Systems Engineering, U.S.A.: Springer, 2009.

[9] J. S. Li, S. M. Meerkov and L. Zhang, "Production systems engineering problem, solutions and applications," Annual Reviews in Control, vol. 34, pp. 73-88, 2010.

[10] C. M. Kwon and S. G. Lim, "Bottleneck detection based on duration of active periods," Journal of the Korea Society for Simulation, vol. 22, pp. 35-41, 2013.

[11] L. Li, Q. Chang, G. X. Xiao, and S. Ambani, "Throughput bottleneck prediction of manufacturing systems using time series analysis," Journal of Manufacturing Science and Engineering, vol. 133, 2011.
[12] G. Wang, S. Song, Y.W. Shin, and D. H. Moon, “A simulation based study on increasing production capacity in a crankshaft line considering limited budget and space,” Journal of Korean Institute of Industrial Engineers, vol. 40, pp.481-491, 2014.

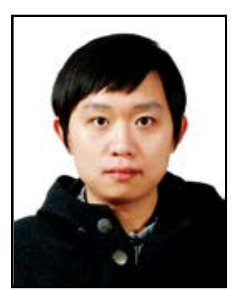

Guan Wang received B.S. at the Department of Management Engineering of Hebei University of Technology in Tianjin, China, in 2008. He got M.Sc. and Ph.D. at the Department of Industrial and Systems Engineering of Changwon National University, Korea in 2010 and 2014, respectively. He is currently a lecturer at the School of Management and Economics of Hebei University of Science and Technology in Shijiazhuang City, Hebei Province, China PR since 2015. Dr. Wang's interests are in manufacturing system design, discrete event simulation and 3D mechanical simulation for automation.

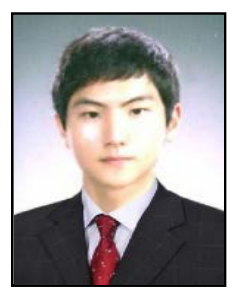

Dae Ryoung Lee received B.S. at the Department of Industrial and Systems Engineering of Changwon National University, Korea, in 2014. He is a graduate student at the Department of Eco-friendly Marine Plant FEED Engineering of Changwon National University. Mr. Lee's interests are in production scheduling and simulation modeling, especially developing scheduler based on simulation model.

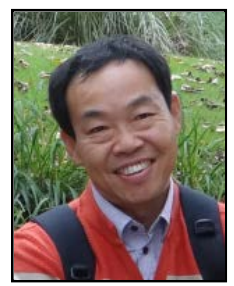

Yang Woo Shin received B.S. from Kyungpook National University, and M.Sc. and Ph.D. in the Department of Mathematics at KAIST (Korea Advanced Institute of Science and Technology). He is currently a professor at the Department of Statistics of Changwon National University in Korea since 1991. Prof. Shin's research interests include probability modeling, queueing theory and its applications.

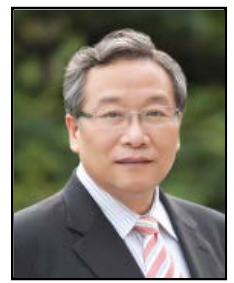

Dug Hee Moon received B.S. from Hanyang University, and M.Sc. and Ph.D. in the Department of Industrial Engineering at KAIST (Korea Advanced Institute of Science and Technology). He is currently a professor at the Department of Industrial and Systems Engineering of Changwon National University in Korea since 1990. Prof. Moon's research interests are in manufacturing system design, factory layout design, scheduling, supply chain management and simulation in practice such as automotive, electrical appliance, aircraft and weapon industries. 